Baslan and his colleagues started out by studying copy number variation because this method requires less starting material than is needed for full sequencing - and single cells don't have much DNA to begin with. In March, however, scientists from the BGI genomics center in Shenzhen, China reported a way to amplify the DNA three-million-fold, creating enough starting material to decode the entire protein-coding portion of the genome down to the single nucleotide level for individual cancer cells (Cell 148, 873-885, 2012).

Future single-cell sequencing projects should also become easier thanks to the creation last month of a new single-cell genomics center at the Broad Institute in Cambridge, Massachusetts, with equipment and staff support from Fluidigm Corporation, a microfluidics technology company based in South San Francisco. According to Fluidigm's president and chief executive Gajus Worthington, the new center will begin by focusing on cancer stem cells and circulating tumor cells. "We hope that studying these cells will lead to a new type of biopsy that identifies from the start whether a tumor will spread or is drug resistant," he says.

Jeanne Erdmann

monoclonal antibody directed against the CD20 protein found on the surface of B cells, results in significant improvements in tumor regression and survival (Leuk. Res. 35, 787-792, 2011). Trials of YM155 in combination with carboplatin plus paclitaxel chemotherapy to treat patients with lung cancer and other solid tumors are ongoing.

Meanwhile, alternative strategies to suppress survivin have also entered early clinical testing, including vaccines to generate an antigenspecific immune response against tumor cells that express survivin and several antisense oligonucleotides. The latter-which include products from Indiana's Eli Lilly, New Jersey's Enzon Pharmaceuticals and Denmark's Santaris Pharma-should in theory be able to silence the BIRC5 gene, which codes for survivin, with more precision than small molecules such as YM155.

Nonetheless, Anthony Tolcher, director of clinical research at South Texas Accelerated Research Therapeutics in San Antonio, who has led trials involving both YM155 and antisense therapies, expects that delivery challenges associated with getting antisense molecules into the target cancer cells will most likely mean that YM155 or another small-molecule drug yet to enter the clinic will be the first such agent to hit the market. Whether or not that drug hits survivin directly, however, remains to be seen.

\section{A history of drugs on the weight list}

Antiobesity medications have a weighty history in the US. Throughout the post-war era, amphetamines were widely touted for their appetite-suppressing effects until their addictive potential prompted the US Food and Drug Administration (FDA) to ban their use as a weight-loss aid in 1979. The mid-1990s then saw the surge in popularity of fen-phen-a combination of the two anorectics, fenfluramine and phenterminebefore that diet pill, too, was pulled from the market due to concerns over serious heart valve problems. More recent attempts haven't fared much better: Meridia, Acomplia and Ephedra all had to be yanked from pharmacy shelves owing to spotty safety records.

Will the latest obesity remedies_Lorqess (lorcaserin) and Qnexa (a combination of phentermine and topiramate)—stand the test of time? In 2010, the FDA, worried about dangerous side effects, rejected both agents. But in February, FDA advisors reviewed new data and recommended granting market approval to Qnexa, a drug from Mountain View, California-based Vivus that combines an appetite suppressant and an antiepileptic, as an obesity treatment. And last month, the same committee ruled in favor of licensing Lorqess, a serotonin receptor agonist from San Diego-based Arena Pharmaceuticals.

If the FDA follows its panel's advice-decisions are expected before mid-July-these drugs would be the first new prescription antiobesity pills to reach the US market in 13 years. Here we give the skinny on the recent checkered past of weight-loss drugs.

Abbott's Meridia (sibutramine), a neurotransmitter reuptake inhibitor, wins FDA approval.

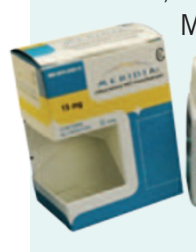

Meridia was withdrawn from the US market in October 2010 after clinical trials revealed an increased risk of heart attack and stroke.

The European Medicines Agency approves Sanofi's cannabinoid receptor blocker Acomplia (rimonabant), a decision the agency reversed in 2009 owing to psychiatric risks. The FDA never approved Acomplia.

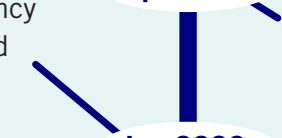

\section{Nov 1997}

Roche's Xenical (orlistat), which reduces intestinal fat absorption by inhibiting pancreatic lipase, receives Apr 1999 FDA approval. It remains the only prescription antiobesity medication approved for long-term use.

The FDA bans the sale of ephedracontaining dietary supplements, citing heart-rate and blood-pressure abnormalities, leading to strokes and sudden deaths.
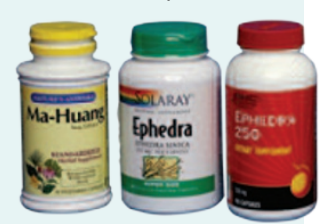

\section{The FDA approves}

GlaxoSmithKline's Alli, an overthe-counter formulation of orlistat.

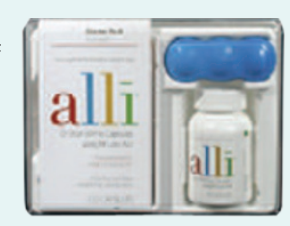

Feb 2007

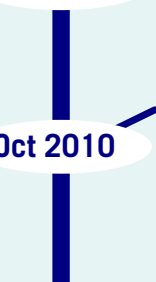

Despite an earlier advisory panel's recommendation in favor of approval, the FDA rejects Orexigen Therapeutics's Contrave, a combination of the antidepressant bupropion and the opioid receptor blocker naltrexone, citing concerns about possible cardiovascular risks.
Feb 2011

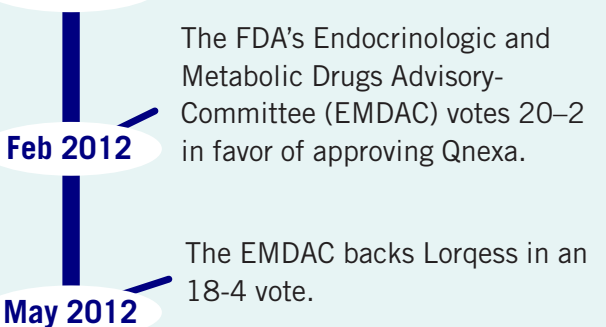

The FDA rejects both Lorqess and Qnexa. Less than two years later, advisory panels recommend in favor of approving both medicines, arguing that the benefits outweigh the risks.

The FDA's Endocrinologic and Metabolic Drugs Advisory-

Committee (EMDAC) votes 20-2

Feb 2012

The EMDAC backs Lorqess in an

May 2012 18-4 vote. 